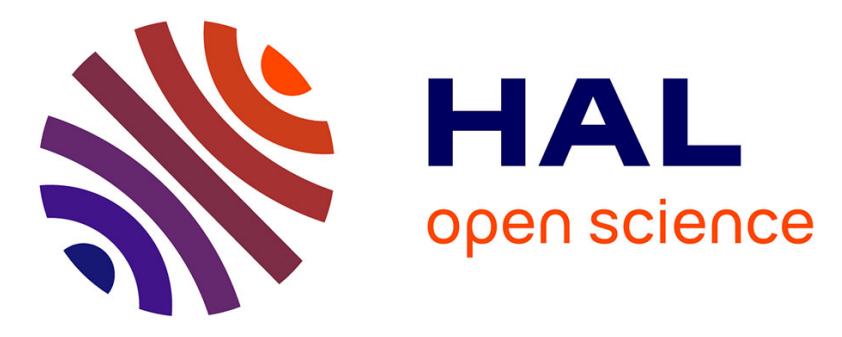

\title{
Control of a cantilever array by Periodic Networks of Resistances
}

\author{
H. Hui, Y. Yakoubi, Michel Lenczner, N. Ratier
}

\section{To cite this version:}

H. Hui, Y. Yakoubi, Michel Lenczner, N. Ratier. Control of a cantilever array by Periodic Networks of Resistances. 11th International Conference on Thermal, Mechanical and Multiphysics Simulation and Experiments in Micro/Nanoelectronics and Systems (EuroSimE 2010), Apr 2010, Bordeaux, France. pp.1-6, 10.1109/ESIME.2010.5464558 . hal-00677432

\section{HAL Id: hal-00677432 \\ https://hal.science/hal-00677432}

Submitted on 14 Apr 2021

HAL is a multi-disciplinary open access archive for the deposit and dissemination of scientific research documents, whether they are published or not. The documents may come from teaching and research institutions in France or abroad, or from public or private research centers.
L'archive ouverte pluridisciplinaire HAL, est destinée au dépôt et à la diffusion de documents scientifiques de niveau recherche, publiés ou non, émanant des établissements d'enseignement et de recherche français ou étrangers, des laboratoires publics ou privés. 


\title{
Control of a Cantilever Array by Periodic Networks of Resistances
}

\author{
H. Hui ${ }^{1,3}$ Y. Yakoubi ${ }^{2}$ M. Lenczner ${ }^{3}$ and N. Ratier ${ }^{3}$ \\ ${ }^{1}$ School of Mechatronic Northwestern Polytechnical University, 127 Youyi Xilu, 710072 Xi' an Shaanxi, China \\ ${ }^{2}$ Laboratoire Jacques-Louis Lions, Université Pierre et Marie Curie, Boîte courrier 187, 75252 Paris Cedex 05, France \\ ${ }^{3}$ FEMTO-ST, Département Temps-Fréquences Université de Franche-Comté, \\ 26, Chemin de l'Epitaphe, 25030 Besançon Cedex, France \\ Email: hui.hui@femto-st.fr, yyakoubi@ann.jussieu.fr, michel.lenczner@utbm.fr and nicolas.ratier@femto-st.fr
}

\begin{abstract}
In this paper, we present a two-scale model including an optimal active control for a one-dimensional cantilever array with regularly spaced actuators and sensors. With the purpose of implementing the control in real time, we propose an approximation that may be realized by an analog distributed electronic circuit. More precisely, our ana$\log$ processor is made by Periodic Network of Resistances (PNR). The control approximation method is based on two general concepts, namely on functions of operators and on the Dunford-Schwartz representation formula. We conducted validations of the control approximation method as well as of its effect in the complete control loop.
\end{abstract}

\section{Introduction}

In the past decade, a number of papers have been focused on semi-decentralized distributed optimal control for systems with distributed actuators and sensors. Most of them are dealing with infinite length systems, see [1] and [10] for systems governed by partial differential equations, and [3] for discrete systems. In the papers [4] and [5] the authors have introduced an approximation of an optimal control to a finite length beam endowed with a periodic distribution of piezoelectric sensors and actuators. Even if it was giving satisfactory results, it was suffering from some limitations. In [9] it has been extended so that to cover a larger range of systems and to increase its precision and robustness. Indeed, the new method does not require that each operator of the state equation and of the cost functional be functions of a same operator but they must be only functions of a same operator up to some change of variable operators. Regarding precision, the Taylor series approximating a function of an operator has been replaced by the use of the Dunford-Schwartz representation formula followed by a quadrature rule for the contour integral.

Here we apply our new method to a recently developed and validated two-scale model of cantilever arrays, submitted in the paper [8]. It is rigorously justified thanks to an adaptation of the two-scale approximation method introduced in [6] and detailed in [7]. Its main advantage is that in the same time it requires little computing effort and it is reasonably precise.

This paper presents results from an implementation of the new semi-decentralized optimal control strategy on the two-scale model of cantilever arrays. We provide results regarding precision and cost. However our calculations have been carried out using the simplest optimal control strategy, namely a Linear Quadratic Regulator. As in [5], we also provide a realization of the semi-decentralized control scheme through a Periodic Network of Resistances (PNR), implementing a finite difference scheme for the partial differential operator in the Dunford-Schwartz formula. Finally, we quote that the entire approach can be extended to other linear optimal control problems, i.e. $L Q G$ or $H_{\infty}$ controls as well as to more physical actuating and sensing principles.

\section{A Two-Scale Model of Cantilever Arrays}

We consider a one-dimensional cantilever array comprised of an elastic base, and a number of clamped elastic cantilevers with free end, see Figure 1. Assuming that the number of cantilevers is sufficiently large, a homogenized model was derived using a two-scale approximation method. This is reported in the detailed paper [7] devoted to static regime. The corresponding model extended to dynamic regime is introduced in the letter [6]. The modelling papers were written in view of Atomic Force Microscopy application.

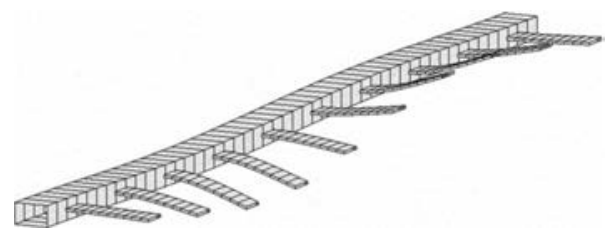

Fig. 1: Array of Cantilevers

After a number of simplifications, the approximate homogenized model expressed in the two-scale referential, which is a rectangle $\Omega=\left(0, L_{B}\right) \times\left(0, L_{C}^{*}\right)$. The parameters $L_{B}$ and $L_{C}^{*}$ represent respectively the base length in the macroscale $x$-direction and the scaled cantilever length in the microscale $y$-direction. The base is modelled by the line $\Gamma=\left\{(x, y) \mid x \in\left(0, L_{B}\right)\right.$ and $\left.y=0\right\}$, and the rectangle $\Omega$ is filled by an infinite number of cantilevers. We describe the system motion by its bending displacement only. So, the base is governed by an Euler-Bernoulli beam equation with two kinds of distributed forces, one exerted by the attached cantilevers and the other, denoted by $u(t, x, 0)$, originating from an actuator distribution. The bending displacement, the mass per unit length, the bending coefficient and the scaled cantilever width being denoted by $w(t, x, 0)$, $\rho^{B}, R^{B}$ and $\ell_{C}^{*}$, the base governing equation states

$$
\rho^{B} \partial_{t t}^{2} w+R^{B} \partial_{x \cdots x}^{4} w+\ell_{\mathbf{C}}^{*} R^{C} \partial_{y y y}^{3} w=u \text { in } \Gamma .
$$

The base is assumed to be clamped, so the boundary con- 
ditions are

$$
w=\partial_{x} w=0,
$$

at its ends. Each cantilever is oriented in the $y$-direction, and its motion is governed by the Euler-Bernoulli equation distributed along the $y$-direction. It is subjected to a control force $u(t, x, y)$ taken as distributed along each whole cantilever. It can be replaced by any other realistic force distribution. Denoting by $w(t, x, y), \rho^{C}$ and $R^{C}$ cantilever bending displacements, the mass per unit length, and the bending coefficient, the governing equation in $(x, y) \in \Omega$ is

$$
\rho^{C} \partial_{t t}^{2} w+R^{C} \partial_{y \cdots y}^{4} w=u
$$

endowed with the boundary conditions

$$
\begin{cases}\partial_{y} w=0 & \text { at } y=0 \\ \partial_{y y}^{2} w=\partial_{y y y}^{3} w=0 & \text { at } y=L_{C}^{*}\end{cases}
$$

representing an end clamped in the base, and a free end. The weak formulation associated to (1-4) states as

$$
\begin{aligned}
& \left.\int_{0}^{L_{B}}\left(\rho^{B} \partial_{t t}^{2} w v+R^{B} \partial_{x x}^{2} w \partial_{x x}^{2} v\right)\right|_{\Gamma} d x \\
& +l_{C}^{*} \int_{\Omega} \rho^{C} \partial_{t t}^{2} w v+R^{C} \partial_{y y}^{2} w \partial_{y y}^{2} v d y d x \\
& =\left.\int_{0}^{L_{B}}(u v)\right|_{\Gamma} d x+l_{C}^{*} \int_{\Omega} u v d y d x
\end{aligned}
$$

for any regular function $v$, satisfying in particular the conditions: $v=\partial_{x} v=0$ at both end of the base and $\partial_{y} v=0$ at $y=0$ at the junction.

\section{Model Reformulation}

To simplify the model, but keeping its distributed feature, we discretize in the $y$-direction projecting on a basis $K_{n}(y)=\int_{0}^{y} y T_{n}^{\prime}(y) d y$, where $T_{n}(y)$ is the basis of Chebyshev polynomial. We define the approximations of the displacement and of the control

$$
\left\{\begin{aligned}
w(t, x, y) & \approx \sum_{n=1}^{N} w_{n}(t, x) K_{n}(y), \\
u(t, x, y) & \approx \sum_{n=1}^{N} u_{n}(t, x) K_{n}(y),
\end{aligned}\right.
$$

where $w_{n}(t, x)$ and $u_{n}(t, x)$ are the polynomial coefficients in the approximation of $w$ and $u$ respectively. We also choose $v \approx \sum_{m=1}^{N} v_{m}(t, x) K_{m}(y)$, so we find that $\left(w_{n}(t, x)\right)_{n=1,2, \cdots, N}$ are the solutions to a set of equations posed on $\Gamma$,

$$
\begin{aligned}
& \sum_{n, m=1}^{N} M_{m, n} \partial_{t t}^{2} w_{n}+K_{m, n}^{B} \partial_{x \cdots x}^{4} w_{n} \\
& +K_{m, n}^{C} w_{n}=\sum_{n, m=1}^{N} \widetilde{B}_{m, n} u_{n} \text { in }[0, \infty) \times \Gamma .
\end{aligned}
$$

The boundary conditions are $\sum_{n=1}^{N} w_{n}(t, 0) K_{n}(0)=$ $\sum_{n=1}^{N} \partial_{x} w_{n}(t, 0) K_{n}(0)=0$ and $\sum_{n=1}^{N} w_{n}\left(t, L_{B}\right) K_{n}(0)=$
$\sum_{n=1}^{N} \partial_{x} w_{n}\left(t, L_{B}\right) K_{n}(0)=0$. In (6), we use the notations

$$
\begin{aligned}
& M_{m, n}=\left.\rho^{B}\left(K_{m} K_{n}\right)\right|_{\Gamma}+l_{C}^{*} \rho^{C} \int_{0}^{L_{C}^{*}} K_{m} K_{n} d y, \\
& K_{m, n}^{B}=\left.R^{B}\left(K_{m} K_{n}\right)\right|_{\Gamma}, \\
& K_{m, n}^{C}=l_{C}^{*} R^{C} \int_{0}^{L_{C}^{*}} \partial_{y y}^{2} K_{m} \partial_{y y}^{2} K_{n} d y, \\
& \widetilde{B}_{m, n}=\left.\left(K_{m} K_{n}\right)\right|_{\Gamma}+l_{C}^{*} \int_{0}^{L_{C}^{*}} K_{m} K_{n} d y .
\end{aligned}
$$

The $L Q R$ problem is set for control variables $\left(u_{n}\right)_{n=1,2, \cdots, N} \in L^{2}(\Gamma)^{N}$ and for the cost functional

$$
\begin{gathered}
\mathcal{J}=\int_{0}^{+\infty} \sum_{n=1}^{N}\left\|\partial_{x x}^{2} w_{n}(t, x)\right\|_{L^{2}(\Gamma)}^{2} \\
+\left\|u_{n}(t, x)\right\|_{L^{2}(\Gamma)}^{2} d t .
\end{gathered}
$$

The choice of the functional is related to vibration stabilization of the microcantilever array.

\section{Classical Formulation of the $L Q R$ Problem}

Now, we write the above $L Q R$ problem in a classical abstract setting, see [2], even if we do not detail the functional framework. We set $z^{T}=\left(\begin{array}{ll}w_{n} & \partial_{t} w_{n}\end{array}\right)_{n=1,2, \cdots, N}$ the state variable, $u^{T}=\left(u_{n}\right)_{n=1,2, \cdots, N}$ the control variable, $A=\left(\begin{array}{cc}0_{N \times N} & I_{N \times N} \\ -\left(M^{-1}\left(K^{B} \partial_{x}^{4}+K^{C}\right)\right)_{N \times N} & 0_{N \times N}\end{array}\right)$ the state operator, $B=\left(\begin{array}{c}0_{N \times N} \\ \left(M^{-1} \widetilde{B}\right)_{N \times N}\end{array}\right)$ the control operator, $C=\left(\begin{array}{cc}\partial_{x x}^{2} I_{N \times N} & 0_{N \times N} \\ 0_{N \times N} & 0_{N \times N}\end{array}\right)$ the observation operator, and $S=I_{N \times N}$ the weight operator. Consequently, the $L Q R$ problem, consisting in minimizing the functional under the constraint (6), may be written under its usual form as

$$
\begin{aligned}
& \partial_{t} z(t, x)=A z(t)+B u(t) \\
& \text { for } t>0 \text { and } z(0)=z_{0},
\end{aligned}
$$

with the minimized cost functional (7). We know that $(A, B)$ is stabilizable and that $(A, C)$ is detectable, in the sense that the system is controllable and observable. It follows that for each $z_{0}$, the $L Q R$ problem (8) admits a unique solution

$$
u^{*}=-K z
$$

where $K=S^{-1} B^{*} P$, and $P$ is the unique self-adjoint nonnegative solution to the operational Riccati equation

$$
A^{*} P+P A-P B S^{-1} B^{*} P+C^{*} C=0 .
$$

\section{Semi-Decentralized Approximation}

This Section is devoted to formulate the approximation method. The mathematical derivation has been introduced in a paper [9]. We denote by $\Lambda$, the mapping: $\Lambda$ : $f \longrightarrow w$, where $w$ is the unique solution of $\partial_{x \cdots x}^{4} w=f$ in $\Gamma$ with the boundary conditions $w=\partial_{x} w=0$ for $x=\left\{0, L_{B}\right\}$. The spectrum $\sigma(\Lambda)$ is discrete and made up of real eigenvalues $\lambda_{k}$. They are solutions to the eigenvalue problem $\Lambda \phi_{k}=\lambda_{k} \phi_{k}$ with $\left\|\phi_{k}\right\|_{L^{2}(\Gamma)}=1$. In the sequel, $I_{\sigma}=\left(\sigma_{\min }, \sigma_{\max }\right)$ refers to an open interval that includes the complete spectrum. 


\subsection{Factorization of $K$ by a Matrix of Functions of $\Lambda$}

In this part, we introduce the factorization of the controller $K$ under the form of a product of a matrix of functions of $\Lambda$. To do so, we introduce the change of variable operators $\Phi_{Z}=\left(\begin{array}{cc}\Lambda^{\frac{1}{2}} & 0 \\ 0 & I\end{array}\right), \Phi_{U}=I$ and $\Phi_{Y}=\left(\begin{array}{cc}\partial_{x}^{2} \Lambda^{\frac{1}{2}} & 0 \\ 0 & I\end{array}\right)$, from which we introduce the matrices of functions of $\Lambda, a(\Lambda)=\Phi_{Z}^{-1} A \Phi_{Z}, b(\Lambda)=$ $\Phi_{Z}^{-1} B \Phi_{U}, c(\Lambda)=\Phi_{Y}^{-1} C \Phi_{Z}$ and $s(\Lambda)=\Phi_{U}^{-1} S \Phi_{U}$, simple to implement on a semi-decentralized architecture. A straightforward calculation yield

$$
\begin{aligned}
& a(\lambda)=\left(\begin{array}{cc}
0 & I \\
\widetilde{M} & 0
\end{array}\right), b(\lambda)=\left(\begin{array}{c}
0 \\
M^{-1} \widetilde{B}
\end{array}\right), \\
& c(\lambda)=\left(\begin{array}{ll}
I & 0 \\
0 & 0
\end{array}\right), \text { and } s(\lambda)=I,
\end{aligned}
$$

where $\widetilde{M}=-M^{-1}\left(K^{B} \lambda^{-1 / 2}+K^{C} \lambda^{1 / 2}\right)$. From (9), the optimal controller $K$ admits the factorization

$$
K=k(\Lambda)=\Phi_{U} q(\Lambda) \Phi_{Z}^{-1},
$$

where $q(\lambda)=s^{-1}(\lambda) b^{T}(\lambda) p(\lambda)$, and where for all $\lambda \in \sigma, p(\lambda)$ is the unique self-adjoint nonnegative matrix solving the algebraic Riccati equation

$$
\begin{gathered}
a^{T}(\lambda) p+p a(\lambda)-p b(\lambda) s^{-1}(\lambda) b^{T}(\lambda) p \\
+c^{T}(\lambda) c(\lambda)=0 .
\end{gathered}
$$

\subsection{Approximation of the Functions of $\Lambda$}

We build the approximation in two steps. Firstly, we use a rational approximation $k_{R}(\Lambda)$ of $k(\Lambda)$, then it is approximated by another function $k_{R, M}$ which is simple to discretize, and yields an accurate approximation. To do so, we use the Dunford-Schwartz formula, see [12], representing a function of an operator, because it involves only the operator $(\zeta I-\Lambda)^{-1}$ which may be simply and accurately approximated. Since the function $k(\Lambda)$ is not known, the spectrum $\sigma(\Lambda)$ cannot be easily determined, so we approximate $k(\lambda)$ by a highly accurate rational approximation $k_{R}(\Lambda)$, then the Dunford-Schwartz formula is applied to $k_{R}(\Lambda)$ with a path tracing out ellipses including $I_{\sigma}$ but no poles. Since the interval $I_{\sigma}$ is bounded, for each function $k_{i j}(\lambda)$ have a rational approximation over $I_{\sigma}$, we write under a global formulation, (which may be understood component wise)

$$
k_{R}(\lambda)=\frac{\sum_{m=0}^{R^{N}} d_{m} \lambda^{m}}{\sum_{m^{\prime}=0}^{R^{D}} d_{m^{\prime}}^{\prime} \lambda^{m^{\prime}}},
$$

where $d_{m}, d_{m^{\prime}}^{\prime}$ are matrices of coefficients and $R=$ $\left(R^{N}, R^{D}\right)$ is the couple comprised of the matrices $R^{N}$ of numerator polynomial degrees and the matrices $R^{D}$ of denominator polynomial degrees. The path $\mathcal{C}$, in the DunfordSchwartz formula,

$$
k_{R}(\Lambda)=\frac{1}{2 i \pi} \int_{\mathcal{C}} k_{R}(\zeta)(\zeta I-\Lambda)^{-1} d \zeta,
$$

is chosen to be an ellipse parameterized by $\zeta(\theta)=$ $\zeta_{1}(\theta)+i \zeta_{2}(\theta)$, with $\theta \in[0,2 \pi]$. The parametrization is used as a change of variable, so the integral can be approximated by a quadrature formula involving $M$ nodes $\left(\theta_{l}\right)_{l=1, \ldots, M} \in[0,2 \pi]$, and $M$ weights $\left(\omega_{l}\right)_{l=1, \ldots, M}$, $I_{M}(g)=\sum_{l=1}^{M} g\left(\theta_{l}\right) \omega_{l}$.

In the following equations, we state that the matrices $k_{R}(\zeta)$ associated to the rational approximation with the numerator polynomial degrees $R^{N}$ and the denominator polynomial degrees $R^{D}$. So, for each $z \in L^{2}(\Gamma)^{2 N}$ and $\zeta \in C$, we introduce the $2 N$-dimensional vector field

$$
v^{\zeta}=-i \zeta^{\prime} k_{R}(\zeta)(\zeta I-\Lambda)^{-1} z .
$$

Decomposing $v^{\zeta}$ into its real part $v_{1}^{\zeta}$ and its imaginary part $v_{2}^{\zeta}$, the couple $\left(v_{1}^{\zeta}, v_{2}^{\zeta}\right)$ is solution of the system

$$
\left\{\begin{array}{l}
\zeta_{1} v_{1}^{\zeta}-\zeta_{2} v_{2}^{\zeta}-\Lambda v_{1}^{\zeta}=\operatorname{Re}\left(-i \zeta^{\prime} k_{R}(\zeta)\right) z \\
\zeta_{2} v_{1}^{\zeta}+\zeta_{1} v_{2}^{\zeta}-\Lambda v_{2}^{\zeta}=\operatorname{Im}\left(-i \zeta^{\prime} k_{R}(\zeta)\right) z
\end{array}\right.
$$

Thus, combining the rational approximation $k_{R}$ and the quadrature formula yields an approximate realization $k_{R, M}(\Lambda)$ of $k(\Lambda)$,

$$
k_{R, M}(\Lambda) z=\frac{1}{2 \pi} \sum_{l=1}^{M} v_{1}^{\zeta\left(\theta_{l}\right)} \omega_{l} .
$$

This formula is central in the method, so it is the center of our attention in the simulations. A fundamental remark is that, a "real-time" realization, $k_{R, M}(\Lambda) z$, requires solving $M$ systems like (13) corresponding to the $M$ quadrature nodes $\zeta\left(\theta_{l}\right)$. The matrices $k_{R}\left(\zeta\left(\theta_{l}\right)\right)$ could be computed "off-line" once and for all, and stored in memory, so their determination would not penalize a rapid real-time computation. In total, the ultimate parameter responsible of accuracy in a real-time computation, apart from spatial discretization discussed in next Section, is $M$ the number of quadrature points.

\section{Circuit Implementation}

To realize an optimal control by a set of distributed circuits, we introduce a spatial discretization and synthesis of Equation (13). The interval $\Gamma$ is meshed with regularly spaced nodes separated by a distance $h$, we introduce $\Lambda_{h}^{-1}$ the finite difference discretization of $\Lambda^{-1}$, associated with the clamping boundary condition. In practice, the discretization length $h$ is chosen small compared to the distance between cantilevers. Then, $z_{h}$ denoting the vector of nodal values of $z$, for each $\zeta$ we introduce $\left(v_{1, h}^{\zeta}, v_{2, h}^{\zeta}\right)$, a discrete approximation of $\left(v_{1}^{\zeta}, v_{2}^{\zeta}\right)$, solution of the discrete set of equations,

$$
\begin{aligned}
& \zeta_{1} v_{1, h}^{\zeta}-\zeta_{2} v_{2, h}^{\zeta}-\Lambda_{h} v_{1, h}^{\zeta}=\operatorname{Re}\left(-i \zeta^{\prime} k_{R}(\zeta)\right) z_{h}, \\
& \zeta_{2} v_{1, h}^{\zeta}+\zeta_{1} v_{2, h}^{\zeta}-\Lambda_{h} v_{2, h}^{\zeta}=\operatorname{Im}\left(-i \zeta^{\prime} k_{R}(\zeta)\right) z_{h} .
\end{aligned}
$$

Finally, an approximate optimal control, intended to be implemented in a set of spatially distributed actuators, could be estimated from the nodal values,

$$
k_{R, M, h} z_{h}=\frac{1}{2 \pi} \sum_{l=1}^{M} v_{1, h}^{\zeta_{l}} \omega_{l}
$$




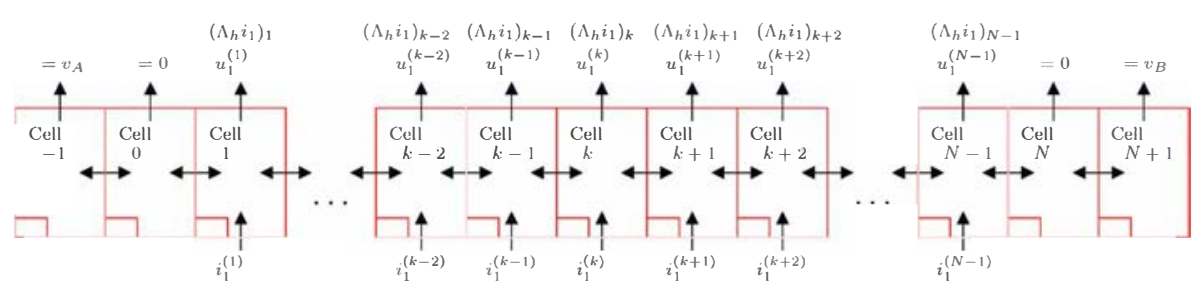

Fig. 2: Analog computation of $\Lambda_{h} v_{1}$.

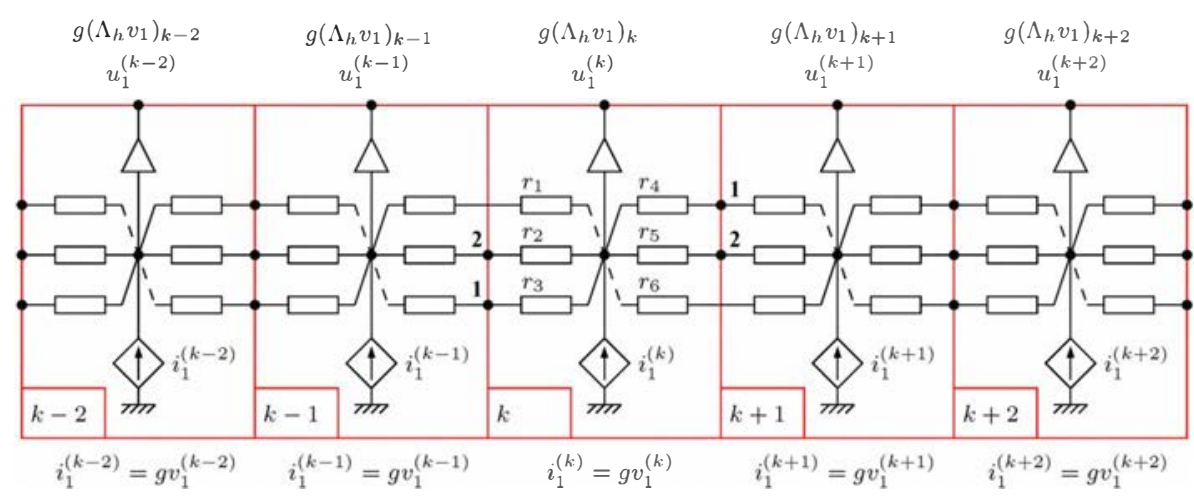

Fig. 3: Five adjacent interior cells.

estimated at mesh nodes in the following. We propose a synthesis of (15-16) by a distributed electronic circuit. The system is rewritten under the manageable form (1718) and for the sake of simplicity, we use the notations $\alpha=$ $\operatorname{Re}\left(-i \zeta^{\prime} k_{R}(\zeta)\right) z_{h}, \beta=\operatorname{Im}\left(-i \zeta^{\prime} k_{R}(\zeta)\right) z_{h}, v_{1}=v_{1, h}^{\zeta}$, and $v_{2}=v_{2, h}^{\zeta}$.

$$
\begin{aligned}
& v_{1}=\frac{\zeta_{1}}{\zeta_{1}^{2}+\zeta_{2}^{2}}\left(\alpha+\Lambda_{h} v_{1}\right)+\frac{\zeta_{2}}{\zeta_{1}^{2}+\zeta_{2}^{2}}\left(\beta+\Lambda_{h} v_{2}\right) \\
& v_{2}=\frac{\zeta_{1}}{\zeta_{1}^{2}+\zeta_{2}^{2}}\left(\beta+\Lambda_{h} v_{2}\right)-\frac{\zeta_{2}}{\zeta_{1}^{2}+\zeta_{2}^{2}}\left(\alpha+\Lambda_{h} v_{1}\right)
\end{aligned}
$$

\subsection{Analog computation of $\Lambda_{h} v_{1}$ and $\Lambda_{h} v_{2}$}

The analog computation of $\Lambda_{h} v_{1}$ and $\Lambda_{h} v_{2}$ are made by Periodic Network of Resistances(PNR) circuits [11]. These electronic circuits have been developed to solve a large class of PDEs by analog computation. More exactly, PNR circuits compute the finite difference solution of a PDE.

PNR circuits are gathering of cells (Figure 2), the interior cells are indexed by $k=1, \ldots, N-1$, while the boundary cells correspond to $k=-1,0, N$ and $N+1$. We will show that the circuits solve the equations $\Lambda_{h}^{-1} u_{1}=i_{1}$. If the current sources $i_{1}$ are replaced by a voltage controlled current sources defined by $i_{1}=g v_{1}$ (with $g$ is a real number), the voltage outputs of the circuits $u_{1}$ solve $g\left(\Lambda_{h} v_{1}\right)$ and so $\Lambda_{h} v_{1}$. The computation of $\Lambda_{h} v_{2}$ is done in the same way.

The interior cell $k$ which compute $\left(\Lambda_{h} v_{1}\right)_{k}$ is represented on Figure 3 with its two adjacent cells on each side.
We call $\rho_{1}$ the resistances between the potentials $u_{1}^{(k)}$ and $u_{1}^{(k \pm 2)}$, and $\rho_{2}$ the resistances between the potentials $u_{1}^{(k)}$ and $u_{1}^{(k \pm 1)}$. By applying the Kirchhoff Current Law (KCL) at node $u_{1}^{(k)}$, rearranging some terms and dividing by $h^{4}$, the equation of the cell $k$ can be written under the form:

$$
\begin{array}{r}
\frac{1}{h^{4}}\left[-\frac{1}{\rho_{1}} u_{1}^{(k-2)}-\frac{1}{\rho_{2}} u_{1}^{(k-1)}+2 u_{1}^{(k)}\left(\frac{1}{\rho_{1}}+\frac{1}{\rho_{2}}\right)\right. \\
\left.-\frac{1}{\rho_{2}} u_{1}^{(k+1)}-\frac{1}{\rho_{1}} u_{1}^{(k+2)}\right]=\frac{1}{h^{4}} i_{1}^{(k)}
\end{array}
$$

If one choose the negative potential $\rho_{1}=-h^{4} \rho_{0}$ and positive potential $\rho_{2}=h^{4} \rho_{0} / 4$, then the potential at node $u_{1}^{(k)}$ is expressed as a function of its neighbor voltages as

$$
\begin{gathered}
\frac{1}{h^{4}}\left[u_{1}^{(k-2)}-4 u_{1}^{(k-1)}+6 u_{1}^{(k)}-4 u_{1}^{(k+1)}\right. \\
\left.+u_{1}^{(k+2)}\right]=\rho_{0} i_{1}^{(k)}
\end{gathered}
$$

which is the stencil of the differential operation $\Lambda^{-1}$. Consequently, the whole electronic circuit composed of $N-1$ cells computes the finite difference approximation $u_{1}=$ $\Lambda_{h} i_{1}=g\left(\Lambda_{h} v_{1}\right)$. The numerical value of $\rho_{0}$ only changes the magnitude of the voltages $u_{1}^{(k)}$. The values of the resistances inside a cell depend only on the circuit topology and are easily expressed as a function of $\rho_{1}$ or $\rho_{2}$, here the resistances of the cells can be taken as $r_{1}=r_{3}=r_{4}=$ $r_{6}=\rho_{1} / 4$ and $r_{2}=r_{5}=\rho_{2} / 2$.

The VCCS (Voltage Controlled Current Source) $i_{1}^{(k)}$ of Figure 3 is controlled by the voltage $v_{1}^{(k)}$ through the equation $i_{1}^{(k)}=g v_{1}^{(k)}$. The four boundary cells are represented in Figure 4. The imposed values of the voltages correspond 

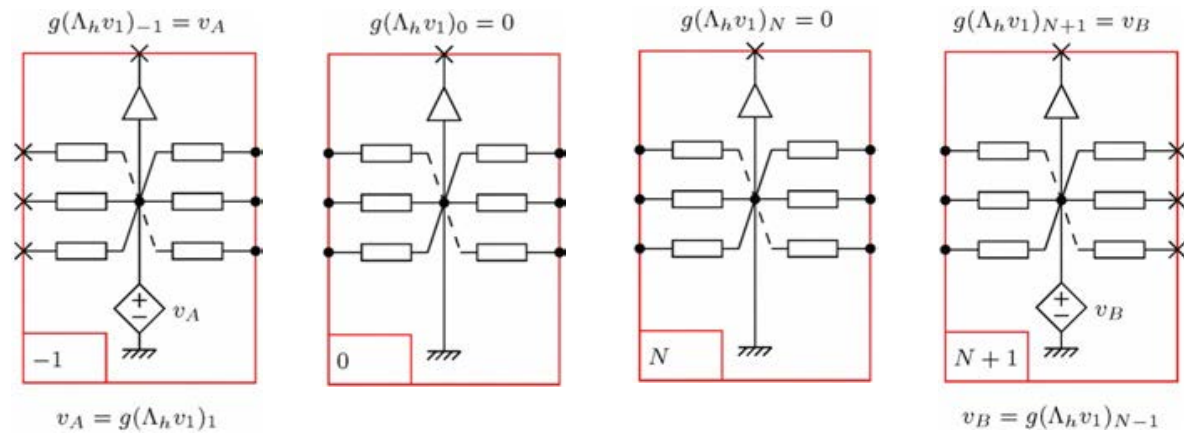

$v_{B}=g\left(\Lambda_{h} v_{1}\right)_{N-1}$

Fig. 4: Four boundary cells.

to the clamping boundary condition. Remark that the terminals denoted by a cross are not connected, so the resistances are linked by one side at them can be removed without changing the behavior of the circuits. They are saved to show clearly the real difference between interior cells and boundary cells.

\subsection{Analog computation of equation (17)}

The analog computation of Equation (17) can made by an array of classical non inverting summing amplifiers of Figure 5. Notice that there is no current exchange between these circuits and PNR inputs and outputs, see buffers in Figure 3. Analysis of the circuit of Figure 5 leads to (19).

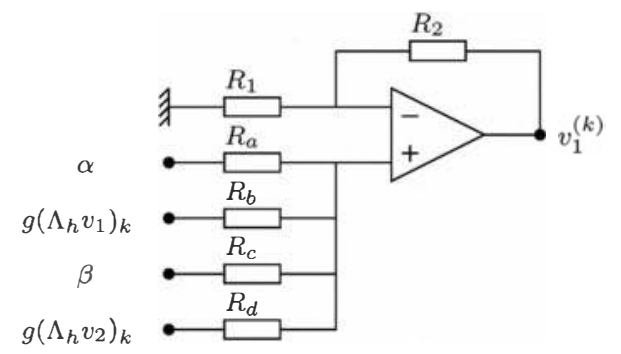

Fig. 5: Analog computation of the $k$-th equation (17).

With a proper choice of resistances, Figure 5 solve (17),

$$
\begin{gathered}
v_{1}^{(k)}=\frac{R_{1}+R_{2}}{R_{1}}\left[\frac{R_{u}}{R_{a}} \alpha+\frac{R_{u}}{R_{b}} g\left(\Lambda_{h} v_{1}\right)_{k}\right. \\
\left.+\frac{R_{u}}{R_{c}} \beta+\frac{R_{u}}{R_{d}} g\left(\Lambda_{h} v_{2}\right)_{k}\right],
\end{gathered}
$$

where $\frac{1}{R_{u}}=\frac{1}{R_{a}}+\frac{1}{R_{b}}+\frac{1}{R_{c}}+\frac{1}{R_{d}}$.

\subsection{Analog computation of equation (18)}

In a very similar way, the analog computation of Equation 18 can made by an array of classical difference summing amplifiers of Figure 6. Analysis of the circuit of Figure 6 leads to (20). With a proper choice of resistances, Figure 6 solve (18),

$$
\begin{aligned}
v_{2}^{(k)}= & \frac{R_{v}}{R_{w}} \frac{R_{2}^{\prime}}{R_{a}^{\prime}} \beta+\frac{R_{v}}{R_{w}} \frac{R_{2}^{\prime}}{R_{b}^{\prime}} g\left(\Lambda_{h} v_{2}\right)_{k} \\
& -\frac{R_{2}^{\prime}}{R_{c}^{\prime}} \alpha-\frac{R_{2}^{\prime}}{R_{d}^{\prime}} g\left(\Lambda_{h} v_{1}\right)_{k},
\end{aligned}
$$

where $\frac{1}{R_{v}}=\frac{1}{R_{a}^{\prime}}+\frac{1}{R_{b}^{\prime}}+\frac{1}{R_{1}^{\prime}}$ and $\frac{1}{R_{w}}=\frac{1}{R_{c}^{\prime}}+\frac{1}{R_{d}^{\prime}}+\frac{1}{R_{2}^{\prime}}$.

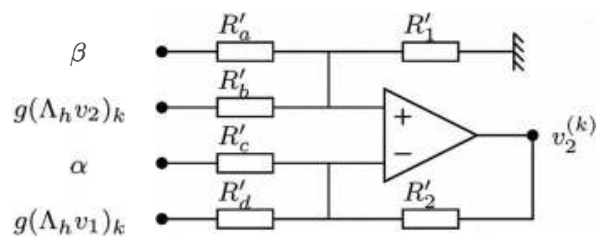

Fig. 6: Analog computation of the $k$-th equation (18).

\section{Numerical Simulation}

In this Section, we validate the approximation method, established in Section 5, by a numerical simulation. We consider a silicon array comprised of an elastic base clamped of 10 elastic cantilevers, with base dimensions $L_{B} \times l_{B} \times h_{B}=500 \mu m \times 16.7 \mu m \times 10 \mu m$, and one cantilever dimensions $L_{C} \times l_{C} \times h_{C}=41.7 \mu m \times 12.5 \mu m \times$ $1.25 \mu \mathrm{m}$. The model parameters of base and cantilever: the bending coefficient $R^{B}=1.09 \times 10^{-5} \mathrm{~N} / \mathrm{m}, R^{C}=2.13 \times$ $10^{-4} \mathrm{~N} / \mathrm{m}$ the mass per unit length $\rho^{B}=0.0233 \mathrm{~kg} / \mathrm{m}$, $\rho^{C}=0.00291 \mathrm{~kg} / \mathrm{m}$. In the rational approximation, the numerator polynomial degrees $R^{N}$ and the denominator polynomial degrees $R^{D}$ can be chosen sufficiently large (namely $R^{N}=R^{D}=20$ ) so that the relative errors between the exact solution $k$ and its rational approximation $k_{R}, e=\frac{\left\|k_{R}-k\right\|_{L^{2}\left(I_{\sigma}\right)}}{\|k\|_{L^{2}\left(I_{\sigma}\right)}}$, can be in the order of $10^{-8}$. This choice has no effect on real-time computation time.

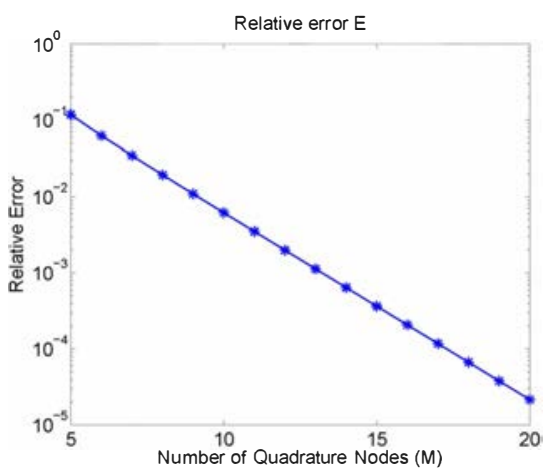

Fig. 7: The relative error between the exact solution and the final approximation 
Numerical integrations have been performed with a standard trapezoidal quadrature rule. The relative error, $E=\frac{\left\|k_{R}, M-k\right\|_{L^{2}\left(I_{\sigma}\right)}}{\|k\|_{L^{2}\left(I_{\sigma}\right)}}$, between the exact control function and final approximation are shown in Figure 7, for the number of nodes $M$ varying from 5 to 20 . It may be easily tuned without changing spatial complexity associated with the finite difference discretization of $\Lambda^{-1}$.

We also present the ratio of the computation time of solving the whole system for varying number of nodes $M$ to the reference computation time of solving the whole system for $M=20$, see Figure 8 .

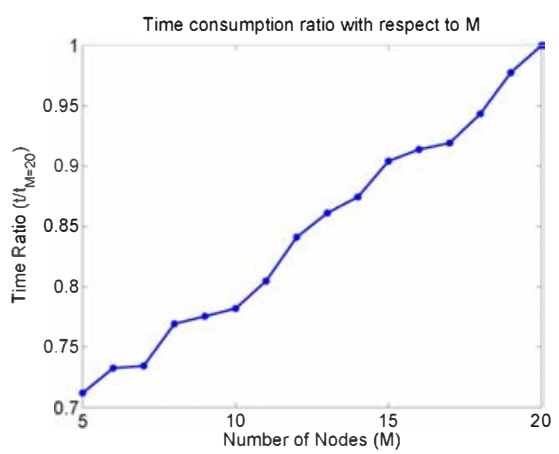

Fig. 8: The ration of computation time

\section{Conclusion}

In this paper, we have presented a semi-decentralized approximation of an optimal control operator applied to a two-scale model of microcantilever arrays. This model is discretized in $y$-direction projecting on a transformed basis of Chebyshev polynomials. It has been shown that the semi-decentralized optimal controller can be implemented by a set of distributed electronic circuits. Numerical simulations have been carried out to validate the method and study its performances. This method can be extended to other optimal control theories, such as $L Q G$ or $H_{\infty}$.

\section{References}

1. B. Bamieh, F. Paganini, and M.Dahleh. Distributed control of spatially invariant systems. IEEE Trans actions on Automatic Control, 47(7):1091-1107, 2002.

2. R. F. Curtain and H. Zwart. An introduction to infinite-dimensional linear systems theory. Texts in Applied Mathematics. Springer-Verlag, 1995.

3. R. D'Andrea and G. E. Dullerud. Distributed control design for spatially interconnected systems. IEEE Trans. Automat. Control, 48(9):1478-1495, 2003.

4. M. Kader, M. Lenczner, and Z. Mrcarica. Approximation of an optimal control law using a distributed electronic circuit: application to vibration control. 328(7):547 - 53, 2000.

5. M. Kader, M. Lenczner, and Z. Mrcarica. Distributed optimal control of vibrations: a high frequency approximation approach. 12(3):437 - 446, 2003.
6. M. Lenczner. A multiscale model for atomic force microscope array mechanical behavior. Applied Physics Letters, 90:091908, 2007.

7. M. Lenczner and R. C. Smith. A two-scale model for atomic force microscopes arrays in static operating regime. Maths. and. Compt. Modelling, 46:776805, 2007.

8. Michel Lenczner, Emmanuel Pillet, Scott Cogan, and Hui Hui. A multiscale model of cantilever arrays and its updating. 7:125-135, 2009.

9. Michel Lenczner and Youssef Yakoubi. Semidecentralized approximation of optimal control for partial differential equations in bounded domains. Comptes Rendus Mécanique, 337:245-250, 2009.

10. F. Paganini and B. Bamieh. Decentralization properties of optimal distributed controllers. 2(9):18771882, 1998.

11. Nicolas Ratier. Towards $2 d$ electronic circuits in the spatial domain. Proceedings of the 13th WSEAS international conference on Circuits, pages 212-218, 2009.

12. K. Yosida. Functional analysis. Classics in Mathematics. Springer-Verlag, reprint of the sixth edition edition, 1980. 